\title{
Modelling the evolution of the Sun's open and total magnetic flux
}

\author{
N. A. Krivova ${ }^{1}$, S. K. Solanki ${ }^{1,2}$, B. Hofer ${ }^{1,3}$, C.-J. Wu ${ }^{1}$, I. G. Usoskin ${ }^{4}$, and R. Cameron ${ }^{1}$ \\ 1 Max-Planck-Institut für Sonnensystemforschung, Justus-von-Liebig-Weg 3, Göttingen, Germany \\ e-mail: natalie@mps.mpg.de \\ 2 School of Space Research, Kyung Hee University, Yongin, Gyeonggi-Do 446-701, Republic of Korea \\ 3 Institut für Astrophysik, Georg-August-Universität Göttingen, Friedrich-Hund-Platz 1, 37077 Göttingen, Germany \\ ${ }^{4}$ University of Oulu, Oulu, Finland
}

Received 5 February 2021 / Accepted 25 March 2021

\begin{abstract}
Solar activity in all its varied manifestations is driven by the magnetic field. Two global quantities are particularly important for many purposes, the Sun's total and open magnetic flux, which can be computed from sunspot number records using models. Such sunspotdriven models, however, do not take into account the presence of magnetic flux during grand minima, such as the Maunder minimum. Here we present a major update of a widely used simple model, which now takes into account the observation that the distribution of all magnetic features on the Sun follows a single power law. The exponent of the power law changes over the solar cycle. This allows for the emergence of small-scale magnetic flux even when no sunspots have been present for multiple decades and leads to non-zero total and open magnetic flux also in the deepest grand minima, such as the Maunder minimum, thus overcoming a major shortcoming of the earlier models. The results of the updated model compare well with the available observations and reconstructions of the solar total and open magnetic flux. This opens up the possibility of improved reconstructions of the sunspot number from time series of the cosmogenic isotope production rate.
\end{abstract}

Key words. Sun: activity - Sun: heliosphere - Sun: magnetic fields - Sun: photosphere - solar-terrestrial relations

\section{Introduction}

The precise history of solar activity and its underlying magnetic field is of interest for a number of reasons. Firstly, records of solar activity and of the magnetic field pose an important constraint on models for the enhancement and the evolution of magnetic flux (mainly dynamo models). Secondly, such records are important for understanding the history of the Sun's influence on the Earth (either through changes in its irradiance, or through space weather effects). Thirdly, a long record of solar activity is needed to understand how the Sun compares with other Sunlike stars in its level of activity and variability (e.g., Radick et al. 2018; Reinhold et al. 2020).

Solar activity, in all its diverse manifestations, is driven by its magnetic field, so that knowledge of the history of solar activity implies knowledge of its magnetic field. Two widely used quantities describing the global magnetic field of the Sun are the global open and unsigned total magnetic flux. They are, for example, used in heliospheric physics, for the reconstruction of solar irradiance, or as measures of solar activity when comparing the Sun with other stars. These quantities, being global in nature, can be reconstructed from more indirect proxies of solar activity and magnetism, such as the sunspot number and concentrations of the cosmogenic isotopes ${ }^{14} \mathrm{C}$ or ${ }^{10} \mathrm{Be}$ in terrestrial archives.

A first model to compute the solar open flux from the sunspot number was developed by Solanki et al. (2000), based on a simple differential equation describing the evolution of the open flux, $\phi_{\text {open }}$. In spite of its simplicity, it successfully reproduced the empirically-reconstructed evolution of the open flux by Lockwood et al. (1999) and the ${ }^{10} \mathrm{Be}$ concentration in ice cores (Beer et al. 1990). This simple model was extended by
Solanki et al. (2002) to also cover the total unsigned magnetic flux, $\phi_{\text {total }}$, but requiring the solution of a set of coupled differential equations to describe the evolution of ephemeral regions (ERs) besides that of active regions (ARs) and of the open flux. All three components of magnetic flux contribute to the evolution of the total magnetic flux. ARs are the large bipolar structures that harbour sunspots at least part of the time, whereas ERs are smaller bipolar regions without sunspots.

The ability of this model to reproduce concentrations of cosmogenic isotopes turned out to be particularly useful (e.g., Usoskin et al. 2002). Although far more sophisticated models have, in the meantime, become available to compute not just global magnetic quantities, but also, for instance, the underlying spatial distribution of the magnetic flux and the detailed input from individual emerging ARs, the very simplicity of this set of models allowed them to be inverted (e.g., Lockwood 2003), so that, for example, the sunspot number could be reconstructed from measured concentrations of cosmogenic isotopes (e.g., Usoskin et al. 2003, 2004, 2016; Solanki et al. 2004; Wu et al. 2018b). The model of Solanki et al. (2002) was further extended and combined with the successful SATIRE model (Spectral And Total Irradiance REconstruction; Fligge et al. 2000; Krivova et al. 2003, 2011) to compute total solar irradiance over the last 400 years (Krivova et al. 2007, 2010). Vieira \& Solanki (2010, hereafter VS2010) have further refined the model by distinguishing between the short-lived and longlived components of the open flux (Ikhsanov \& Ivanov 1999; Cranmer 2002; Crooker et al. 2002; see Vieira \& Solanki 2010 for details), which led to an improved reconstruction of the open flux that displayed a better agreement with observations. This model, with some tuning, has been the basis for further reconstructions of solar spectral irradiance over the telescope 
era (Krivova et al. 2010), as well as the sunspot number and TSI over the Holocene (Vieira et al. 2011; Wu et al. 2018b,a).

One shortcoming of earlier versions of the model discussed above is that the open flux during a grand minimum, such as the Maunder minimum, that is to say during a long period essentially without sunspots, invariably drops to zero. This is because in this model, the emergence rate of the magnetic field on the solar surface is linearly linked to the sunspot number, so that by design during a grand minimum no magnetic flux is allowed to emerge. This leads to a zero open and total flux during the grand minima. It has been shown, however, that signals of solar activity and variability were also present during the Maunder minimum (Beer et al. 1998; Fligge et al. 1999; Usoskin et al. 2001; Miyahara et al. 2004; Riley et al. 2015). This was also confirmed by modelling (Owens et al. 2012) and points to a need for an improvement of the global total and open magnetic flux model.

Furthermore, more recent solar observations provided new insights into the sources, emergence, and evolution of the solar magnetic flux. Thus, Thornton \& Parnell (2011) have combined observations from various sources and found that the emergence rate of bipolar magnetic regions with fluxes between $10^{16} \mathrm{Mx}$ and $10^{23} \mathrm{Mx}$ follows a single power law with a slope of -2.69 .

Here we present a new, strongly revised version of the VS2010 model that builds on these recent solar observational results, replacing the direct proportionality of ERs and ARs with a more up-to-date approach. It does keep the original differential equations, however, meaning that it is not a completely independent model. As a natural outcome of the model, ERs keep emerging even during a grand minimum when there have been no sunspots for multiple decades. This means that neither the open nor the total magnetic flux drop to zero at any time.

The paper is structured as follows. The data used to constrain and test the new model are briefly introduced in Sect. 2. We describe our model and highlight the changes relative to the older version of the model in Sect. 3. The results of the model are presented in Sect. 4, while we summarise and discuss our findings in Sect. 5, where we also provide an outlook on future applications of the new model.

\section{Data}

The model, detailed in Sect. 3, starts from a sunspot number time series and computes the total and open magnetic fluxes of the Sun therefrom. We therefore require a sunspot series as input to the model. To constrain the free parameters of the model, we compared its output to observations and independent data-based reconstructions of the total and open magnetic fluxes. Finally, to test the output of the model, we considered further independent time series of the reconstructed open magnetic flux.

As input to the model, we used the following sunspot number data sets: (1) the international sunspot number v2.0, referred to hereafter as ISN2.0 (Clette \& Lefèvre 2016), and (2) the group sunspot number, or GSN in short (Hoyt \& Schatten 1998). The ISN2.0 data set was extended back to 1643 by adding the sunspot data during the Maunder minimum by Vaquero et al. (2015) scaled up by the factor 1.67 to match the ISN2.0 definition. Also in the GSN record, the values before 1710 were replaced by the data from Vaquero et al. (2015), without any scaling.

To constrain the output of the model, we made use of observations of the total magnetic flux (see Arge et al. 2002; Wang et al. 2005; Wenzler et al. 2006) derived from synoptic charts produced by the three solar observatories with the longest running regular magnetographic measurements: Wilcox Solar Observatory (WSO), Mount Wilson solar Observatory (MWO), and National Solar Observatory at Kitt Peak (NSO/KP). These data sets have already been used to constrain earlier versions of this model and we used the same versions as employed by Krivova et al. (2007, 2010) and Wu et al. (2018a). To constrain the free parameters of the model, we considered the average over at least two (MWO and WSO over 2002-2009) or all three (1976-2002) records for each Carrington rotation.

Furthermore, to better constrain the free parameters of the model, we also used the empirical reconstruction of the open magnetic flux from the geomagnetic aa-index covering the period from 1845 to 2010 (Lockwood et al. 2014). Finally, the quality of the computed open magnetic flux was tested by comparing it with two other independent data sets (without changing the free parameters of the model): (1) a compilation of spacecraft-based in situ measurements by Owens et al. (2017) since 1998, and (2) a reconstruction by Wu et al. (2018b) from decadal INTCAL13 ${ }^{14} \mathrm{C}$ data covering the Holocene prior to 1900 (Reimer et al. 2013).

\section{Model}

\subsection{Magnetic flux emergence and evolution}

Following the approach by Solanki et al. (2000, 2002) and Vieira \& Solanki (2010), we describe the evolution of the solar total and open magnetic flux by a system of ordinary differential equations. However, instead of distinguishing between ARs and ERs as the sources of fresh magnetic flux at the solar surface, we distinguish between ARs and what we call small-scale emergences (SSEs), that is all emergences with fluxes smaller than those in active regions. These, therefore, combine the flux emerging in the form of ephemeral regions and smaller magnetic bipoles all the way down to internetwork fields. The equations describing the evolution of the different (globally averaged) components of the magnetic flux are:

$$
\begin{aligned}
& \frac{\mathrm{d} \phi_{\mathrm{AR}}}{\mathrm{d} t}=\varepsilon_{\mathrm{AR}}-\frac{\phi_{\mathrm{AR}}}{\tau_{\mathrm{AR}}^{0}}-\frac{\phi_{\mathrm{AR}}}{\tau_{\mathrm{AR}}^{\mathrm{s}}}-\frac{\phi_{\mathrm{AR}}}{\tau_{\mathrm{AR}}^{\mathrm{r}}}, \\
& \frac{\mathrm{d} \phi_{\mathrm{SSE}}}{\mathrm{d} t}=\varepsilon_{\mathrm{SSE}}-\frac{\phi_{\mathrm{SSE}}}{\tau_{\mathrm{SSE}}^{0}}-\frac{\phi_{\mathrm{SSE}}}{\tau_{\mathrm{SSE}}^{\mathrm{s}}}, \\
& \frac{\mathrm{d} \phi_{\mathrm{open}}^{\mathrm{r}}}{\mathrm{d} t}=\frac{\phi_{\mathrm{AR}}}{\tau_{\mathrm{AR}}^{\mathrm{r}}}-\frac{\phi_{\mathrm{open}}^{\mathrm{r}}}{\tau_{\mathrm{open}}^{\mathrm{r}}}, \\
& \phi_{\mathrm{open}}^{\mathrm{d} t}=\frac{\phi_{\mathrm{AR}}}{\tau_{\mathrm{AR}}^{\mathrm{s}}}+\frac{\phi_{\mathrm{SSE}}}{\tau_{\mathrm{SSE}}^{\mathrm{s}}}-\frac{\phi_{\mathrm{open}}^{\mathrm{s}}}{\tau_{\mathrm{open}}^{\mathrm{s}}}, \\
& \phi_{\mathrm{total}}^{\mathrm{r}}=\phi_{\mathrm{AR}}+\phi_{\mathrm{open}}^{\mathrm{s}},
\end{aligned}
$$

Here $\phi_{\mathrm{AR}}, \phi_{\mathrm{SSE}}, \phi_{\mathrm{open}}$, and $\phi_{\text {total }}$ refer to the magnetic flux in ARs and SSEs, as well as the open and total magnetic flux (all magnetic fluxes are global, i.e. referring to their unsigned sum over the entire solar surface). The open flux is divided into rapidly $\left(\phi_{\mathrm{open}}^{\mathrm{r}}\right)$ and slowly $\left(\phi_{\mathrm{open}}^{\mathrm{s}}\right)$ evolving components; $\tau_{\mathrm{AR}}^{0}$, $\tau_{\text {SSE }}^{0}, \tau_{\text {open }}^{\mathrm{r}}$, and $\tau_{\text {open }}^{\mathrm{s}}$ are the decay timescales of ARs, SSEs, rapid open flux, and slow open flux, respectively. The flux transfer timescales from ARs and SSEs to slow open flux are $\tau_{\mathrm{AR}}^{\mathrm{s}}$ and $\tau_{\mathrm{SSE}}^{\mathrm{S}}$, respectively, while the corresponding timescale for the transfer of AR flux to the rapid open flux is $\tau_{\mathrm{AR}}^{\mathrm{r}}$. Readers can 


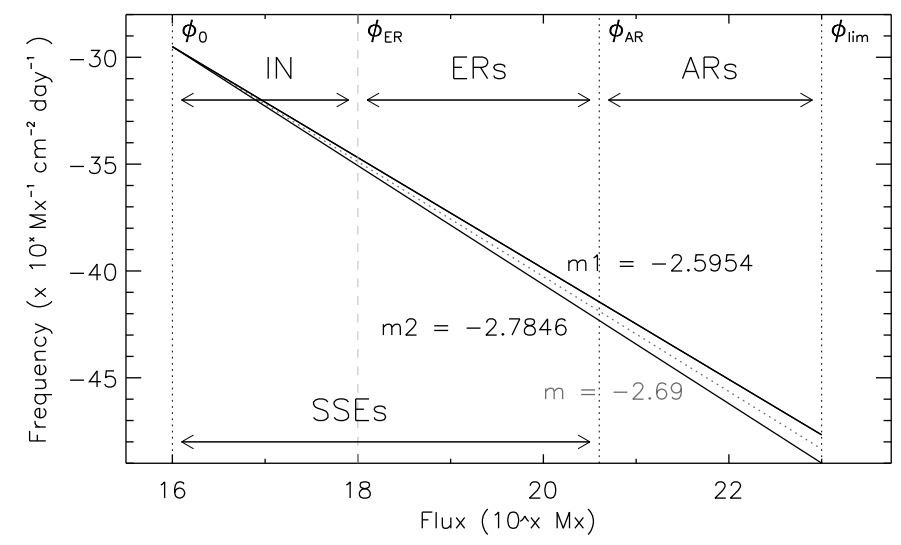

Fig. 1. Frequency of emergence vs. the unsigned flux of an emergence event (see Eq. (9) following Thornton \& Parnell 2011). The values $\phi_{0}, \phi_{\mathrm{ER}}, \phi_{\mathrm{AR}}$, and $\phi_{\text {limit }}$ represent the limit below which the local dynamo flux dominates, the minimum ephemeral region flux, the minimum active region flux, and the upper limit of the active region flux, respectively. The horizontal arrows mark the flux ranges corresponding to the internetwork (IN), ERs, ARs, and SSEs. The SSE range includes IN fields and ERs. The slope $m$ of the distribution was derived by Thornton \& Parnell (2011) by fitting various observations at different activity levels. Slopes $m_{1}$ and $m_{2}$ represent the corresponding distributions at maximum and minimum of solar activity levels for cycle 21 , respectively (see main text for details).

refer to Vieira \& Solanki (2010) for a discussion on the distinction between rapidly and slowly evolving open magnetic flux.

In the original model, the emergence rate of ARs at a given time $t, \varepsilon_{\mathrm{AR}}(t)$, was linked linearly to the sunspot number, $\mathrm{SN}$, at that time:

$\varepsilon_{\mathrm{AR}}(t)=\varepsilon_{\mathrm{AR}}^{\max , 21} \frac{\mathrm{SN}(t)}{\mathrm{SN}^{\max , 21}}$,

where $\varepsilon_{\mathrm{AR}}^{\max , 21}$ and $\mathrm{SN}^{\mathrm{max}, 21}$ are the three-month averaged emergence rate and $\mathrm{SN}$ value observed during the maximum of cycle 21 (taken from Schrijver \& Harvey 1994), respectively. Because at the time that the model was originally developed, large-scale studies of magnetic flux emergence and evolution could not resolve internetwork fields, the earlier model was restricted to ERs being the only magnetic bipoles smaller than ARs. The emergence rate of the ERs, $\varepsilon_{\mathrm{ER}, n}$, of the cycle $n$ was not well known and was assumed to be a sinusoidal function, $g^{n}$, which was stretched such that the length of the ER cycle was longer than the respective sunspot cycle. The amplitude of the ER cycle was simply taken to be proportional to the maximum value of the emergence rate of the ARs of the cycle, so that the emergence rate of ERs over a cycle had the following form:

$\varepsilon_{\mathrm{ER}, n}=\varepsilon_{\mathrm{AR}}^{\max , n} X g^{n}$,

where $X$ is an amplitude factor (a free parameter of the model; same for all cycles). Importantly, Eq. (8) implies a linear relationship between the emergence rate $\varepsilon_{\mathrm{ER}, n}$ and the sunspot number. This, along with the linear relationship between $\varepsilon_{\mathrm{AR}, n}$ and sunspot number (Eq. (7)) results in an absence of flux emergence during extended periods of spotless days.

To overcome this shortcoming, we incorporated more recent solar observations by Thornton \& Parnell (2011) into the model. Using high-resolution Hinode, Solar Optical Telescope/Narrowband Filter Imager (SOT/NFI) observations and combining them with earlier published data, they found that the emergence rate of the magnetic flux on the Sun follows a power-law distribution of

$$
\frac{\mathrm{d} N}{\mathrm{~d} \phi}=\frac{n_{0}}{\phi_{0}}\left(\frac{\phi}{\phi_{0}}\right)^{m}
$$

where $\phi_{0}=10^{16} \mathrm{Mx}$ (the smallest flux per feature that they include in their histograms), $n_{0}=3.14 \times 10^{-14} \mathrm{~cm}^{-2}$ day $^{-1}$ and $m=-2.69$ (see the illustration in Fig. 1).

We note that Thornton \& Parnell (2011) have summarised the results from multiple studies with a wide range of solar activity levels and observing conditions. In earlier studies, Harvey \& Zwaan (1993) and Harvey (1993) found that the emergence rate of ARs varied significantly more between solar activity minimum and maximum than that of ERs. Whereas roughly 8.3 times more ARs emerged during the maximum of cycle 21 than during the minimum (the factor generally grows with the size of the regions, but it was on average about 8.3 for all the ARs they studied), this ratio was roughly two for ERs. The number of the smallest magnetic features, forming the internetwork magnetic fields and having fluxes of $10^{16}-10^{17} \mathrm{Mx}$, appears to be nearly invariable over an activity cycle (Buehler et al. 2013; Lites et al. 2014). These features differ from the larger ones in that they are mainly brought about by a small-scale turbulent dynamo (Vögler \& Schüssler 2007; Rempel 2014) that produces the same amount of magnetic flux nearly independently of largescale activity.

To satisfy these observational constraints, on the one hand, we kept the number of the smallest magnetic features considered here (with a flux per feature of $10^{16} \mathrm{Mx}$ ) fixed at all times. On the other hand, we allowed the exponent $m$ to vary (cf. Parnell et al. 2009; Schrijver \& Harvey 1989) around the empirical value $m=-2.69$ found by Thornton \& Parnell (2011) within the range $m_{1} \geq m \geq m_{2}$, where $m_{1}=m+\Delta m$ and $m_{2}=m-\Delta m$. The slopes $m_{1}$ and $m_{2}$ describe the distributions of emergence rates during periods when the observed sunspot numbers are $\mathrm{SN}_{1}$ and $\mathrm{SN}_{2}$ (with $\mathrm{SN}_{1}>\mathrm{SN}_{2}$ ). In our model, the slope $m$ follows the $\mathrm{SN}, m(\mathrm{SN})$, according to the following non-linear relationship:

$m(\mathrm{SN})=m_{1}-\left(\mathrm{SN}_{1}^{\alpha}-\mathrm{SN}^{\alpha}\right) \frac{m_{1}-m_{2}}{\mathrm{SN}_{1}^{\alpha}-\mathrm{SN}_{2}^{\alpha}}$

where $\alpha$ is a free parameter, fixed by comparing the output of the model to observations and independent reconstructions (see Sect. 3.2).

Now, the emergence rate of magnetic flux in ARs and SSEs at any given time can be calculated as follows:

$\varepsilon_{\mathrm{AR}}=\int_{\phi_{\mathrm{AR}}}^{\phi_{\text {limit }}} \frac{n_{0}}{\phi_{0}}\left(\frac{\phi}{\phi_{0}}\right)^{m} \phi \mathrm{d} \phi=\frac{n_{0}}{(m+2) \phi_{0}{ }^{m+1}}\left(\phi_{\text {limit }}^{m+2}-\phi_{\mathrm{AR}}^{m+2}\right)$

and

$\varepsilon_{\mathrm{SSE}}=\int_{\phi_{0}}^{\phi_{\mathrm{AR}}} \frac{n_{0}}{\phi_{0}}\left(\frac{\phi}{\phi_{0}}\right)^{m} \phi \mathrm{d} \phi=\frac{n_{0}}{(m+2) \phi_{0}{ }^{m+1}}\left(\phi_{\mathrm{AR}}^{m+2}-\phi_{0}^{m+2}\right)$.

Here $\phi_{\mathrm{AR}}=4 \times 10^{20} \mathrm{Mx}$ denotes the magnetic flux of the smallest bipolar regions hosting sunspots, that is to say the smallest active regions (e.g., Zwaan 1978; Schrijver \& Zwaan 2000; van Driel-Gesztelyi \& Green 2015) and $\phi_{\text {limit }}$ is the flux of the largest ARs considered. Since such regions are extremely rare, the exact value of $\phi_{\text {limit }}$ is not important. Following Parnell et al. (2009) and Thornton \& Parnell (2011), we consider $\phi_{\text {limit }}=$ $10^{23} \mathrm{Mx}$, which is somewhat larger than the maximum flux 
Table 1. Parameters of the model.

\begin{tabular}{lccc}
\hline \hline Parameter & ISN & GSN & W18 (ISN) \\
\hline$n_{0}, \mathrm{~cm}^{-2}$ day $^{-1}$ & \multicolumn{2}{c}{$3.14 \times 10^{-14}$} & $\ldots$ \\
$\phi_{0}, \mathrm{Mx}$ & \multicolumn{2}{c}{$10^{16}$} & $\ldots$ \\
$\phi_{\mathrm{AR}}, \mathrm{Mx}$ & \multicolumn{2}{c}{$4 \times 10^{20}$} & $\ldots$ \\
$\phi_{\text {limit }}, \mathrm{Mx}$ & \multicolumn{2}{c}{$10^{23}$} & $\ldots$ \\
$\mathrm{SN}_{1}$ & 217 & 130 & $\ldots$ \\
$\mathrm{SN}_{2}$ & 17 & 10 & $\ldots$ \\
$m$ & \multicolumn{2}{c}{-2.69} & $\ldots$ \\
$\Delta m$ & \multicolumn{2}{c}{0.0946} & $\ldots$ \\
$\tau_{\mathrm{AR}}^{0}, \mathrm{yr}$ & \multicolumn{2}{c}{0.027} & $\ldots$ \\
$\tau_{\mathrm{SSE}}^{0}, \mathrm{yr}$ & $1.1 \times 10^{-5}$ & $\ldots$ \\
\hline$\alpha$ & 0.059 & 0.075 & $\ldots$ \\
$\tau_{\mathrm{open}}^{\mathrm{r}}, \mathrm{yr}$ & 0.16 & 0.09 & 0.14 \\
$\tau_{\mathrm{open}}^{\mathrm{s}}, \mathrm{yr}$ & 3.98 & 3.90 & 3.75 \\
$\tau_{\mathrm{AR}}^{\mathrm{r}}, \mathrm{yr}$ & 2.79 & 1.57 & 2.6 \\
$\tau_{\mathrm{AR}}^{\mathrm{s}}, \mathrm{yr}$ & 88.15 & 89.81 & 88.3 \\
$\tau_{\mathrm{SSE}}^{\mathrm{s}}, \mathrm{yr}$ & 10.15 & 10.11 & $20.6^{(*)}$ \\
\hline
\end{tabular}

Notes. The upper part of the table lists parameters that are fixed, whereas the lower part lists free parameters. For comparison, the last column (W18) lists the values of the free parameters of the old model from the most recent version by Wu et al. (2018a). ${ }^{(*)}$ This value was obtained by Wu et al. (2018a) for ERs rather than SSEs.

$\left(3 \times 10^{22} \mathrm{Mx}\right)$ for ARs listed by Schrijver \& Zwaan (2000) and van Driel-Gesztelyi \& Green (2015). Tests have shown that also the exact value of $\phi_{\mathrm{AR}}$ adopted here plays only a minor role for the end result in the sense that although the free parameters may have slightly different values, the computed open and total magnetic flux remain almost unchanged.

To estimate how the slope $m$ of the distribution given by Eq. (9) changes with activity (Eq. (10)), we rely on the observations by Harvey \& Zwaan (1993) and Harvey (1993) for cycle 21 . They found that the number of emerging ARs in cycle 21 varied between the activity maximum and minimum by a factor of roughly 8.3. The monthly-smoothed sunspot numbers corresponding to these periods (1979-1982 for the maximum, as well as 1975-1976 and 1985-1986 for the preceding and following minima) are then $\mathrm{SN}_{1}=217$ and $\mathrm{SN}_{2}=17$, respectively. These values were obtained for ISN2.0. For GSN, these numbers correspond to $\mathrm{SN}_{1}=130$ and $\mathrm{SN}_{2}=10$. By using the factor of 8.3 found for the emergence frequency of ARs between activity maximum and minimum by Harvey (1993) as a constraint, we obtain $\Delta m=0.0946$ (and thus $m_{1}=-2.5954$ and $\left.m_{2}=-2.7846\right)$.

We note that the values of $m$ reach values higher than $m_{1}$ or lower than $m_{2}$ at times when the sunspot number is higher than $\mathrm{SN}_{1}$ or lower than $\mathrm{SN}_{2}$, respectively. In particular, when the sunspot number is zero, the corresponding $m$ values are $m(0)=-3.952$ for ISN2.0 and $m(0)=-3.677$ for GSN. The value of $m(0)$ is different for ISN2.0 and GSN because the value of $\alpha$, the free parameter of the model (see Table 1, and Sect. 3.2) is different: $\alpha=0.059$ for ISN2.0 and $\alpha=0.075$ for GSN. The largest value of $m$ that the direct sunspot records give was obtained for the peak of cycle 19, with $\max (\mathrm{SN})=370$ (ISN2.0) and 247 (GSN), which results in $m(370)=-2.552$ (ISN2.0) and $m(247)=-2.542(\mathrm{GSN})$. Figure 2a shows the daily (black) and the monthly-smoothed (red) ISN2.0, while the evolution of $m_{\mathrm{SN}}$ computed from the monthly-smoothed ISN for the solar cycle 21 is shown in Fig. 2 b.

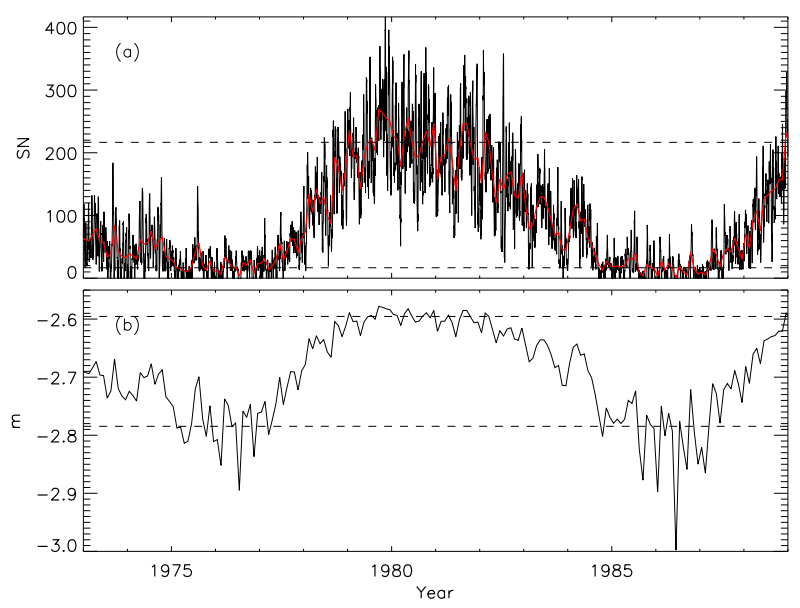

Fig. 2. (a) Daily (black) and 30-day smoothed (red) sunspot number (ISN, v2.0) over cycle 21, including the adjacent mimima. The average values during the activity maximum $\left(\mathrm{SN}_{1}=217\right)$ and minimum $\left(\mathrm{SN}_{2}=17\right)$ of cycle 21 are marked by the horizontal dashed lines. (b) Evolution of the monthly-smoothed power-law slope $m$ over the same period, the horizontal dashed lines mark $m_{1}=-2.5954$ corresponding to $\mathrm{SN}_{1}=217$ and $m_{2}=-2.7846$ corresponding to $\mathrm{SN}_{2}=17$.

\subsection{Parameters of the model}

Our model, described in Sect. 3.1, has a number of parameters, summarised in Table 1. The upper part of the table lists quantities taken or deduced from the literature and kept fixed throughout the analysis, whereas the five free parameters that are allowed to vary when fitting the data sets described in Sect. 2 are given in the lower part. One positive feature of the new model is that it has less free parameters than the old model (the old model required two free parameters to describe the emergence of ERs; see Table 1 of Wu et al. 2018a), giving it less 'wiggle room' to reproduce observational data.

All parameters relevant to the emergence rates of ARs and SSEs (Eqs. (9)-(12)) have been described in the previous section. Here we additionally comment on the decay and transfer times of the various components of the magnetic flux used in the ordinary differential equations describing the flux evolution (Eqs. (1)-(6)).

The decay times of the ARs and SSEs, $\tau_{\mathrm{AR}}^{0}$ and $\tau_{\mathrm{SSE}}^{0}$, were estimated using the observations by Parnell et al. (2009) and Thornton \& Parnell (2011). Whereas Thornton \& Parnell (2011) have analysed the emergence rate of different features as a function of their flux, Parnell et al. (2009) analysed the magnetic flux for all (i.e. instantaneously) observed features. By dividing the total number of the features of a given flux observed at a given instance by their emergence rate, we arrive at their mean lifetime. The lifetime of the features increases with their sizes or fluxes. For the purposes of our study, we made a simplification and calculated the lifetimes of ARs and SSEs as averages over all regions with fluxes above and below $\phi_{\text {AR }}$, respectively, thus obtaining $\tau_{\mathrm{AR}}^{0} \approx 10$ days and $\tau_{\mathrm{SSE}}^{0} \approx 6$ minutes. Since the features with the smallest flux dominate the power law distribution, $\tau_{\mathrm{AR}}^{0}$ is short compared with lifetimes of large ARs and it is closer to lifetimes of small ARs (e.g., Table 1 of the review by van Driel-Gesztelyi \& Green 2015). For the same reason, the SSE lifetime is close to that of internetwork elements, rather than of ERs. For a better comparison with observations of ERs, we therefore introduced $\phi_{\mathrm{ER}}=10^{18} \mathrm{Mx}$ (see Fig. 1), which denotes roughly the lowest magnetic flux contained within ERs. If we now consider only the regions with $\phi_{\mathrm{ER}}<\phi<\phi_{\mathrm{AR}}$ as ERs, then we obtain a lifetime of $\tau_{\mathrm{ER}}^{0} \approx 2$ hours, which 


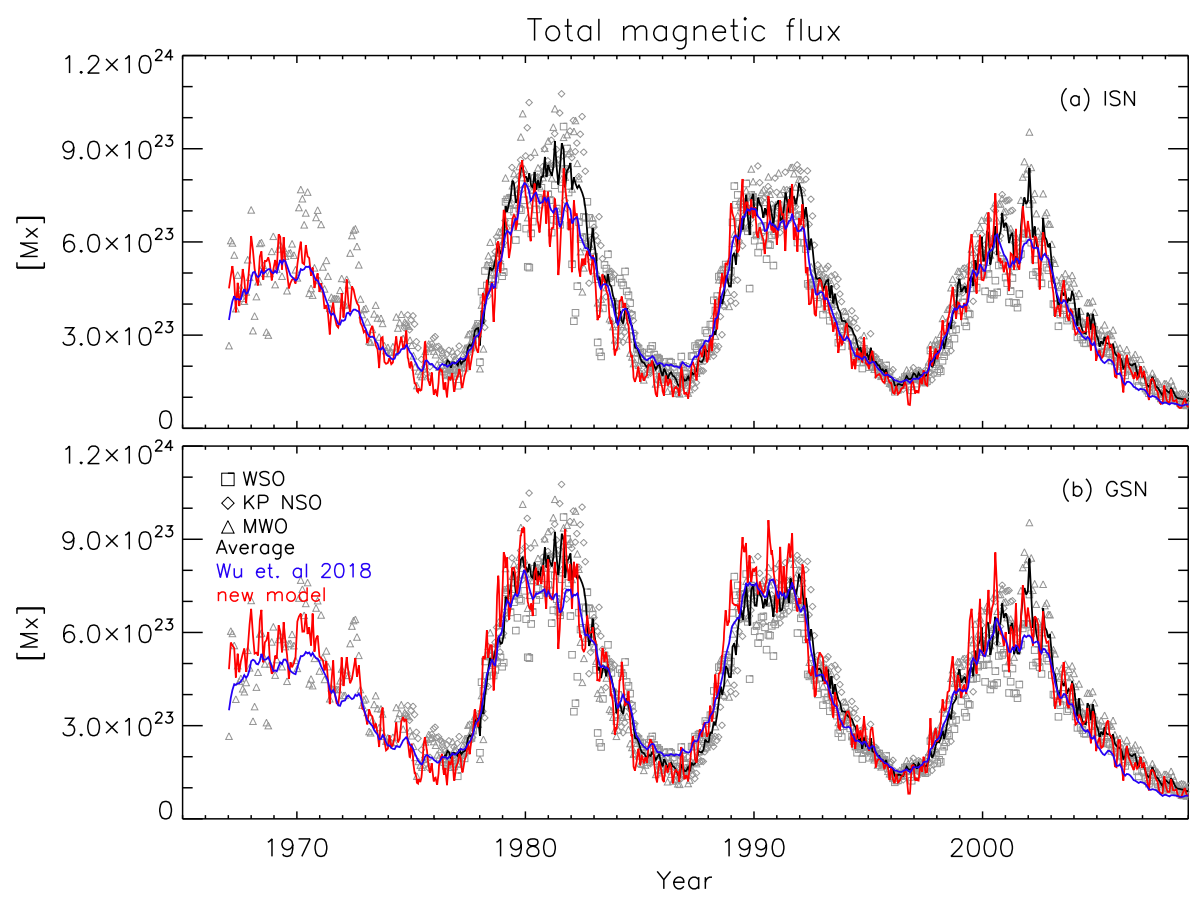

Fig. 3. Evolution of the total magnetic flux over cycles 21-24 computed with the new (red) and old (blue) models, as well as the observed flux. For observations, symbols show individual Carrington rotations and observatories, as indicated in the legend, and solid black line shows their average. For the models, means of the daily total magnetic flux over the Carrington rotations are shown, and the contribution of the SSEs is reduced by a factor of 0.4 (see text for details). Panels $a, b$ : use ISN and GSN as input, respectively. is comparable to the lifetimes of roughly hours to a day for regions with fluxes between $3 \times 10^{18} \mathrm{Mx}$ and $10^{20} \mathrm{Mx}$ listed by van Driel-Gesztelyi \& Green (2015). The maximum to minimum change in the flux emerging in ERs (i.e. with fluxes $\phi_{\mathrm{ER}}<$ $\left.\phi<\phi_{\mathrm{AR}}\right)$ in our model is roughly a factor of 2.5 , which is consistent with the results by Harvey (1993) and Harvey \& Zwaan (1993). We stress that this distinction in ERs and internetwork fields is only used for comparison purposes. Within the model, they are not distinguished (see Sect. 3.1).

The decay timescales of the rapid, $\tau_{\text {open }}^{\mathrm{r}}$, and slow, $\tau_{\text {open }}^{\mathrm{s}}$, open flux, as well as timescales for the flux transfer from ARs and SSEs to slow open flux, $\tau_{\mathrm{AR}}^{\mathrm{s}}$ and $\tau_{\mathrm{SSE}}^{\mathrm{s}}$, respectively, and the timescale for the transfer of the AR flux to the rapid open flux, $\tau_{\mathrm{AR}}^{\mathrm{r}}$, are free parameters of the model (together with $\alpha$ governing the change in the slope $m$ with the $\mathrm{SN}$, as described in Sect. 3.1). These parameters were fixed by comparing the total and open magnetic flux with the corresponding observations listed in Sect. 2.

To do this, we used the genetic algorithm PIKAIA (Charbonneau 1995), which searches for the set of parameters minimising the difference between the modelled and the reference data sets. We minimised the sum of the reduced chi-squared values, $\chi^{2}$, taking the errors of the observations and the number of data points into account. In other words, we searched for the maximum of $1 /\left(\chi_{\text {total }}^{2}+\chi_{\mathrm{OMF}}^{2}\right)$; readers can refer to Vieira \& Solanki (2010) for details and Dasi-Espuig et al. (2016) for a discussion on the uncertainties in the parameter fitting. The best-fit values of the parameters are listed in Table 1.

For comparison, the last column of Table 1 also lists the values of the five free parameters that are already present in the VS2010 model, as obtained by Wu et al. (2018a) for the most recent version of the model. This version used the same ISN2.0 input record extended back with the data from Vaquero et al. (2015) as done here. The values of the parameters are very close in the two models, except $\tau_{\mathrm{SSE}}^{\mathrm{s}}$, for which Wu et al. (2018a) obtained 20.6 years compared to our 10.15 years (10.11 years when using GSN). We note, however, that Wu et al. (2018a) considered ERs rather than SSEs. Interestingly, the values we obtain are close to the value of 10.08 years found to produce a best-fit by Vieira \& Solanki (2010) and lie within the range of 10-90 years that is consistent with observations (see Vieira \& Solanki 2010).

The value of about 4 years for the decay time of the slow open flux $\tau_{\text {open }}^{\mathrm{s}}$ is close to the radial decay term with a timescale of 5 years introduced into surface flux transport simulations by Schrijver et al. (2002) and Baumann et al. (2006) to act on the unipolar fields at the solar poles. This was needed to reproduce the observed polar field reversals. Similarly, the $\tau_{\text {open }}^{\mathrm{r}}$ obtained here (Table 1) is consistent with the estimate of the decay of closed flux carried by interplanetary coronal mass ejections, between 1 and $5 \mathrm{AU}$ of between 38 and 55 days by Owens \& Crooker (2006).

\section{Reconstruction of the total and open magnetic flux}

The computed total magnetic flux from 1965 onward is plotted in Fig. 3. Following Krivova et al. (2007), to account for the flux undetected due to the limited spatial resolution of observations (see Krivova \& Solanki 2004), the contribution of the ER flux was multiplied by a factor of 0.4 before adding it to the contribution from the ARs and the open flux. The means over the daily values for each Carrington rotation are shown. The new and old models are depicted in red and blue, respectively. Symbols show the observations: Wilcox Solar Observatory (WSO, squares), National Solar Observatory at Kitt Peak (NSO KP, diamonds), and Mount Wilson Observatory (MWO, triangles). Each individual symbol represents the total photospheric magnetic flux over a given Carrington rotation. To compute the $\chi^{2}$ value, we used the average of the three (1976-2002) or two (after 2002, see Sect. 2) data sets indicated by the solid black line.

The computed open magnetic flux is shown in Fig. 4. The new model is shown in red, the old model is in blue, and the reconstruction from the geomagnetic aa-index by Lockwood et al. (2014) is represented by the green line. 


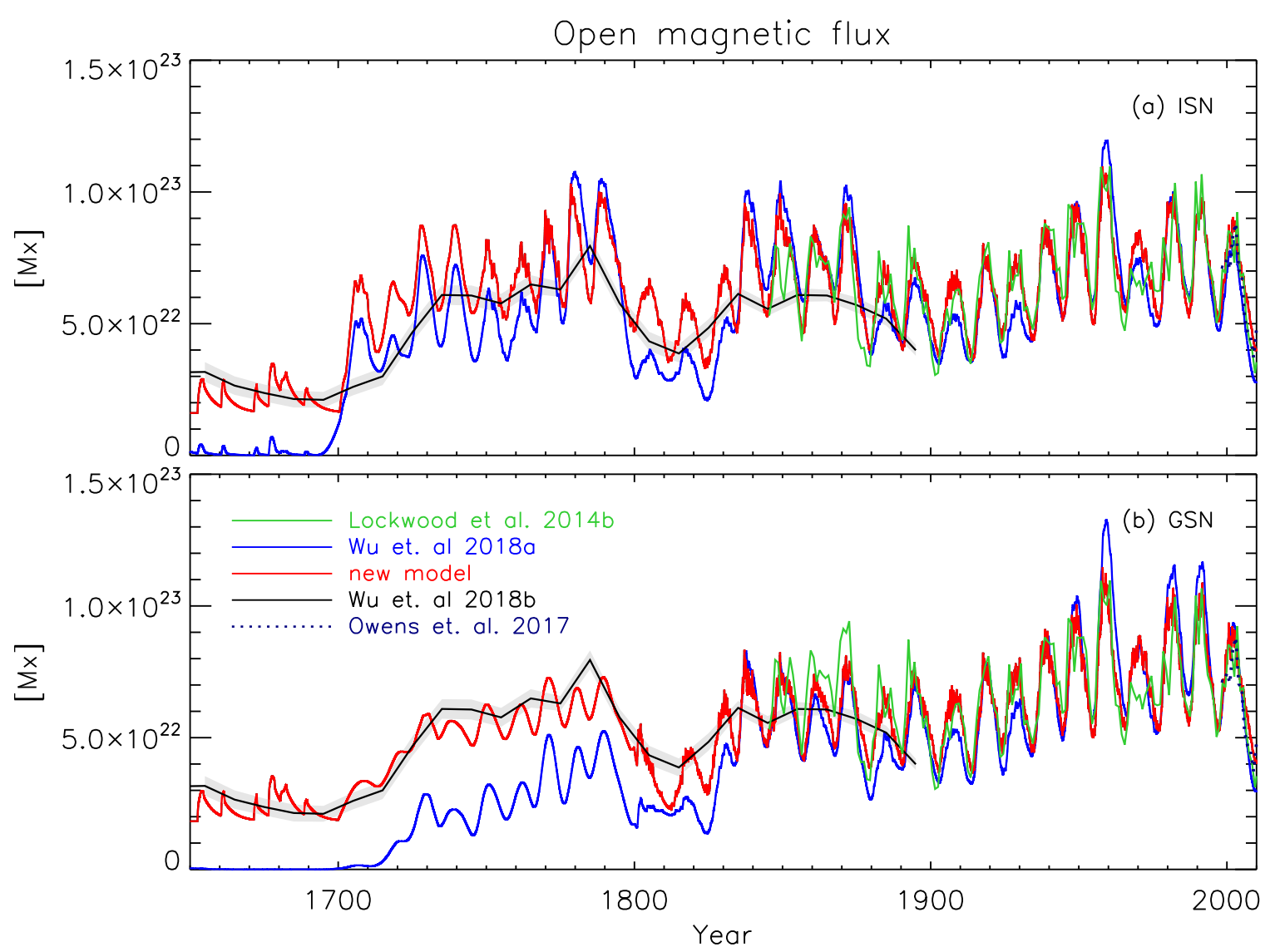

Fig. 4. Open magnetic flux computed with the new (red) and old (blue) models, together with the empirical reconstruction from the geomagnetic aa-index (Lockwood et al. 2014, green). Also shown are the reconstruction by Wu et al. (2018b, green) from the decadal INTCAL13 ${ }^{14} \mathrm{C}$ data (Reimer et al. 2013) and the in situ measurements from Owens et al. (2017, dotted blue line) since 1998. Panels a,b: use ISN and GSN as input, respectively.

Also shown are the in situ measurements by Owens et al. (2017, dotted blue line) since 1998 and a reconstruction (solid black line with shading indicating the uncertainty) of the open flux by Wu et al. (2018b) from independent decadal INTCAL13 ${ }^{14} \mathrm{C}$ data (Reimer et al. 2013). We note that the underlying ${ }^{14} \mathrm{C}$ data and thus also the OF reconstructed from them are decadal averages. The agreement between our model and the ${ }^{14} \mathrm{C}$-based reconstruction is quite good. Particularly impressive is the agreement in the level of the open flux during the Maunder minimum, which is where we expect to see the biggest improvement relative to the old model. We emphasise that this ${ }^{14} \mathrm{C}$-based record was not used to constrain our model. We note that in the old model, the GSN record was used without the data from Vaquero et al. (2015) over the Maunder minimum, and the computed open flux in the old model is therefore essentially flat at the zero level. Interestingly, over the 19th and the first half of the 18th centuries, the GSN-based reconstruction was closer to the isotope-based open flux than the reconstruction from the ISN, which lied somewhat higher.

A quantitative comparison of the total and open magnetic fluxes resulting from the old and the new models with the observations and independent reconstructions is presented in Table 2. The table lists the relative difference in means and the $\chi^{2}$ values (in brackets) between the models and the data. For the total magnetic flux, the results are quite similar for both versions of the model. In both cases, the mean modelled total magnetic flux is somewhat closer to the observations when the GSN is used as input. The absolute difference in the means is slightly higher or lower for the new model if ISN2.0 or GSN are used, respectively. The new $\chi^{2}$ values are somewhat higher than in the old model. This is, however, primarily due to fact that, by model design, the variability of the ER component on time scales shorter than the solar cycle in the old model was essentially smoothed out (see Eqs. (7)-(8)) resulting in weaker short-term fluctuations than in the new model (see Fig. 4). Thus, if we smooth the total flux from both models with a 3 -months window before comparing them, the $\chi^{2}$ values for the old model remain almost unchanged ( 0.036 for both ISN2.0 and GSN), while those for the new model decrease to 0.043 for ISN2.0 and 0.047 for GSN. In all cases, the $\chi^{2}$ values are quite low.

For the open flux, the new model provides a notably better fit than the old model to all three alternative data sets. In all but one case, the absolute mean differences are significantly lower for the new model. The only exception is the GSN-based reconstruction versus the in situ data by Owens et al. (2017), for which the absolute mean difference is slightly lower for the old model. However, the results are quite close for both versions of the model in this case. We also note that these data cover only a short recent period of time, over which the two models do not differ significantly. The $\chi^{2}$ values are lower for the new model in all cases. The fit is poorest for the reconstruction based on the decadal values of ${ }^{14} \mathrm{C}$. Very recently, new ${ }^{14} \mathrm{C}$-based activity measures with annual resolution were published by Brehm et al. (2021). An application of our model to the data set of Brehm et al. (2021) will be the subject of a separate publication (Usoskin et al. 2021). 
N. A. Krivova et al.: Modelling the evolution of the Sun's open and total magnetic flux

Table 2. Comparison of the modelled open and total magnetic fluxes to observations and independent reconstructions, quantified through their relative differences in means (in \%) and reduced $\chi^{2}$ values (listed in brackets).

\begin{tabular}{lccccc}
\hline \hline \multirow{2}{*}{$\begin{array}{l}\text { Input } \\
\text { Model version }\end{array}$} & \multicolumn{2}{c}{ Old } & & \multicolumn{2}{c}{ GSN } \\
\cline { 2 - 3 } \cline { 5 - 6 } & $-7.7(0.037)$ & $-9.9(0.058)$ & & $-4.1(0.037)$ & $0.8(0.058)$ \\
$F_{\text {total }}$ & $-2.2(0.297)$ & $0.4(0.176)$ & & $-4.6(0.389)$ & $-1.6(0.219)$ \\
$F_{\text {open,L14 }}$ & $-15.1(1.718)$ & $6.1(0.630)$ & & $-48.5(1.674)$ & $-9.6(0.230)$ \\
$F_{\text {open,Wu18b }}{ }^{(*)}$ & $-15.4(0.655)$ & $3.8(0.236)$ & & $-3.5(0.495)$ & $5.8(0.252)$ \\
$F_{\text {open,O17 }}$ & &
\end{tabular}

Notes. The top part of the table lists the data sets that were used for parameter fitting (the average of the total magnetic flux measurements and the open flux reconstruction by Lockwood et al. 2014), while the bottom part lists independent data sets that were not used for the optimisation (OF reconstruction from ${ }^{14} \mathrm{C}$ data by $\mathrm{Wu}$ et al. $2018 \mathrm{~b}$ and the in situ measurements by Owens et al. 2017). ${ }^{(*)}$ For decadally-averaged reconstructions. $\mathrm{As}^{14} \mathrm{C}$ data that were used for the reconstruction by Wu et al. (2018b) are decadal averages, only decadally-averaged values of the OF could be reconstructed. Thus, to compute the corresponding $\chi^{2}$ values, our reconstructions were also re-sampled.

\section{Summary and discussion}

We have revised the simple model describing the evolution of the Sun's global total and open magnetic flux, originally proposed by Solanki et al. $(2000,2002)$ and improved by Vieira \& Solanki (2010). The new version of the model takes into account the observation that fluxes of magnetic features follow a single power law, including internetwork fields, ERs and ARs (Parnell et al. 2009; cf. Anusha et al. 2017). It also takes into account the fact that emergence rates of magnetic bipoles with fluxes between $10^{16} \mathrm{Mx}$ and $10^{23} \mathrm{Mx}$, that is from the smallest ERs (and large internetwork features) to the largest ARs, also follow a power law according to the analysis by Thornton \& Parnell (2011).

We assume that the difference in emergence rates between the maximum and the minimum of solar activity is adequately described by the varying power-law exponent, affecting magnetic features with fluxes $\phi>10^{16} \mathrm{Mx}$. Thus, for the smallest features, there is no change in the emergence rate over the solar cycle, while the ratio of emergence rates during maximum to minimum increases steadily with increasing magnetic flux. Thus, the number of emerging ARs varies between the solar maximum and minimum of cycle 21 by a factor of 8.3 , while this factor is 2.5 for ERs and close to unity for internetwork fields. These values are consistent with the respective ratios found by Harvey \& Zwaan (1993) and Harvey (1993), while the fact that the flux in internetwork fields hardly changes over the cycle is in agreement with the results obtained by Buehler et al. (2013) and Lites et al. (2014), for example.

Using the sunspot number time series as input, the model returns time series of the open and total magnetic flux of the Sun. These resulting time series reproduce the open magnetic flux between 1845 and 2010 reconstructed by Lockwood et al. (2014) and the total magnetic flux averaged over individual Carrington rotations obtained by various observatories between 1976 and 2009.

The main novel feature of the results of the model is that, in contrast to the earlier versions of the model, the output open magnetic flux does not drop to essentially zero during the Maunder minimum when almost no sunspots were present for multiple decades, which is in agreement with open flux reconstructed from ${ }^{14} \mathrm{C}$ data (e.g., Wu et al. 2018b). This significant improvement is a result of the model allowing for a non-zero emergence of magnetic flux in small-scale bipolar features (encompassing ERs and internetwork fields) even during extended periods when sunspots were not present, for example, during the Maunder minimum and other, similar grand minima (e.g., Usoskin et al. 2007).

Even with this major update, the model still has some room for further improvements. Because it uses sunspot numbers as input (i.e. the only data of solar activity available prior to the middle of the 19th century), it cannot properly treat variations in solar activity that are not reflected in the number of sunspots. This is particularly evident during grand minima. During such times, sunspots are only occasionally visible, whereas cosmogenic isotopes continue to display cyclic variations. This suggests that, in the context of the present model, the slope $m$ continues to vary in a cyclical manner and can go lower than the lowest value we have obtained $(m(0)=-3.952$ for ISN2.0 and $m(0)=-3.677$ for GSN).

In the old version of the model, the ER emergence was constructed as a smooth, sinusoidal function. In the new model, the ER (and internetwork) emergence rate closely follows that of sunspots and therefore has the same temporal resolution as the input sunspot number series. As a consequence, the new model does not feature temporal lags or shifts to the corresponding sunspot cycle, as in the old model. However, it does account for the finding by Harvey (1994) that small-scale features (in her study, ERs) belonging to a given cycle start emerging at a relatively high rate well before the sunspot cycle starts. The internetwork is independent of sunspot emergence and is, thus, not associated with a particular sunspot cycle. These regions are simply a result of the dynamo not having completely switched off even at times when there are no visible sunspots. In this way, the overlap between neighbouring solar magnetic cycles is naturally introduced. This was the main feature of the original model of Solanki et al. (2000) responsible for the change in the level of the Sun's open magnetic flux from one solar minimum to another, first noticed by Lockwood et al. (1999). At the same time, the new model allows for the dynamo to continue working and produce activity cycles during grand minima, which are sufficiently strong to modulate cosmic rays (see, e.g., Fig. 4 for the evolution of the open flux during the Maunder minimum) and hence influence the production of cosmogenic isotopes (Beer et al. 1998; Fligge et al. 1999; Usoskin et al. 2001), but they are too weak to produce more than occasional sunspots.

The higher level of the magnetic flux during periods of very low solar activity (e.g., during the Maunder and the Dalton minima, see Fig. 4) will presumably lead to a weaker secular variation in the total solar irradiance (TSI) and in particular the rise in the TSI since the Maunder minimum (cf., Yeo et al. 2020), an important parameter for understanding the influence of solar irradiance variability on long-term climate 
trends (e.g., Gray et al. 2010; Solanki et al. 2013). The influence of the revised magnetic flux time series on TSI will be the subject of a future investigation.

Another important application of this model will be in reconstructing total magnetic flux and sunspot numbers from production rates of cosmogenic isotopes. Such an application will require the model to be inverted, as has successfully been done with the older version of the model (e.g., Usoskin et al. 2003, 2004, 2016; Solanki et al. 2004; Wu et al. 2018b). Such work is ongoing and will be the subject of a follow-on publication.

Acknowledgements. We thank Mathew Owens for the helpful comments and stimulating discussion. N.A.K. and S.K.S. acknowledge support by the German Federal Ministry of Education and Research (Project No. 01LG1909C) S.K.S. received support from the Ministry of Education of Korea through the BK21 plus program of the National Research Foundation. B.H. was supported by the International Max-Planck Research School (IMPRS) for Solar System Science at the University of Göttingen. IU acknowledges a partial support from the Academy of Finland (Projects ESPERA no. 321882).

\section{References}

Anusha, L. S., Solanki, S. K., Hirzberger, J., \& Feller, A. 2017, A\&A, 598, A47 Arge, C. N., Hildner, E., Pizzo, V. J., \& Harvey, J. W. 2002, J. Geophys. Res (Space Phys.), 107, 1319

Baumann, I., Schmitt, D., \& Schüssler, M. 2006, A\&A, 446, 307

Beer, J., Blinov, A., Bonani, G., et al. 1990, Nature, 347, 164

Beer, J., Tobias, S., \& Weiss, N. 1998, Sol. Phys., 181, 237

Brehm, N., Bayliss, A., Christl, M., et al. 2021, Nat. Geosci., 14, 10

Buehler, D., Lagg, A., \& Solanki, S. K. 2013, A\&A, 555, A33

Charbonneau, P. 1995, ApJS, 101, 309

Clette, F., \& Lefèvre, L. 2016, Sol. Phys., 291, 2629

Cranmer, S. R. 2002, Space Sci. Rev., 101, 229

Crooker, N. U., Gosling, J. T., \& Kahler, S. W. 2002, J. Geophys. Res. (Space Phys.), 107, 1028

Dasi-Espuig, M., Jiang, J., Krivova, N. A., et al. 2016, A\&A, 590, A63

Fligge, M., Solanki, S. K., \& Beer, J. 1999, A\&A, 346, 313

Fligge, M., Solanki, S. K., \& Unruh, Y. C. 2000, A\&A, 353, 380

Gray, L. J., Beer, J., Geller, M., et al. 2010, Rev. Geophys., 48, RG4001

Harvey, K. L. 1993, PhD Thesis, Univ. Utrecht

Harvey, K. L. 1994, in NATO Advanced Study Institute (ASI) Series C, eds.

R. J. Rutten, \& C. J. Schrijver, Sol. Surf. Magn., 433, 347

Harvey, K. L., \& Zwaan, C. 1993, Sol. Phys., 148, 85

Hoyt, D. V., \& Schatten, K. H. 1998, Sol. Phys., 181, 491

Ikhsanov, R. N., \& Ivanov, V. G. 1999, Sol. Phys., 188, 245

Krivova, N. A., \& Solanki, S. K. 2004, A\&A, 417, 1125

Krivova, N. A., Solanki, S. K., Fligge, M., \& Unruh, Y. C. 2003, A\&A, 399, L1

Krivova, N. A., Balmaceda, L., \& Solanki, S. K. 2007, A\&A, 467, 335

Krivova, N. A., Vieira, L. E. A., \& Solanki, S. K. 2010, J. Geophys. Res., 115, A12112

Krivova, N. A., Solanki, S. K., \& Unruh, Y. C. 2011, J. Atm. Sol.-Terr. Phys., 73, 223
Lites, B. W., Centeno, R., \& McIntosh, S. W. 2014, PASJ, 66, S4 Lockwood, M. 2003, J. Geophys. Res. (Space Phys.), 108, 1128 Lockwood, M., Stamper, R., \& Wild, M. N. 1999, Nature, 399, 437 Lockwood, M., Nevanlinna, H., Barnard, L., et al. 2014, Ann. Geophys., 32, 383 Miyahara, H., Masuda, K., Muraki, Y., et al. 2004, Sol. Phys., 224, 317 Owens, M. J., \& Crooker, N. U. 2006, J. Geophys. Res. (Space Phys.), 111, A10104

Owens, M. J., Usoskin, I., \& Lockwood, M. 2012, Geophys. Res. Lett., 39, L19102

Owens, M. J., Lockwood, M., Riley, P., \& Linker, J. 2017, J. Geophys. Res. (Space Phys.), 122, 10,980

Parnell, C. E., DeForest, C. E., Hagenaar, H. J., et al. 2009, ApJ, 698, 75

Radick, R. R., Lockwood, G. W., Henry, G. W., Hall, J. C., \& Pevtsov, A. A. 2018, ApJ, 855, 75

Reimer, P. J., Bard, E., Bayliss, A., et al. 2013, Radiocarbon, 55, 1869

Reinhold, T., Shapiro, A. I., Solanki, S. K., et al. 2020, Science, 368, 518

Rempel, M. 2014, ApJ, 789, 132

Riley, P., Lionello, R., Linker, J. A., et al. 2015, ApJ, 802, 105

Schrijver, C. J., \& Harvey, K. L. 1989, ApJ, 343, 481

Schrijver, C. J., \& Harvey, K. L. 1994, Sol. Phys., 150, 1

Schrijver, C. J., \& Zwaan, C. 2000, Solar and Stellar Magnetic Activity (Cambridge Univ. Press)

Schrijver, C. J., De Rosa, M. L., \& Title, A. M. 2002, ApJ, 577, 1006

Solanki, S. K., Schüssler, M., \& Fligge, M. 2000, Nature, 408, 445

Solanki, S. K., Schüssler, M., \& Fligge, M. 2002, A\&A, 383, 706

Solanki, S. K., Usoskin, I. G., Kromer, B., Schüssler, M., \& Beer, J. 2004, Nature, 431, 1084

Solanki, S. K., Krivova, N. A., \& Haigh, J. D. 2013, ARA\&A, 51, 311

Thornton, L. M., \& Parnell, C. E. 2011, Sol. Phys., 269, 13

Usoskin, I. G., Mursula, K., \& Kovaltsov, G. A. 2001, J. Geophys. Res., 106, 16039

Usoskin, I. G., Mursula, K., Solanki, S. K., Schüssler, M., \& Kovaltsov, G. A. 2002, J. Geophys. Res., 107, 1374

Usoskin, I. G., Solanki, S. K., Schüssler, M., Mursula, K., \& Alanko, K. 2003, Phys. Rev. Lett., 91

Usoskin, I. G., Mursula, K., Solanki, S., Schüssler, M., \& Alanko, K. 2004, A\&A, 413, 745

Usoskin, I. G., Solanki, S. K., \& Kovaltsov, G. A. 2007, A\&A, 471, 301

Usoskin, I. G., Gallet, Y., Lopes, F., Kovaltsov, G. A., \& Hulot, G. 2016, A\&A, 587, A 150

Usoskin, I. G., Solanki, S. K., Krivova, N., et al. 2021, A\&A, 649, A141

van Driel-Gesztelyi, L., \& Green, L. M. 2015, Liv. Rev. Sol. Phys., 12, 1

Vaquero, J. M., Kovaltsov, G. A., Usoskin, I. G., Carrasco, V. M. S., \& Gallego, M. C. 2015, A\&A, 577, A71

Vieira, L. E. A., \& Solanki, S. K. 2010, A\&A, 509, A100

Vieira, L. E. A., Solanki, S. K., Krivova, N. A., \& Usoskin, I. 2011, A\&A, 531, A6

Vögler, A., \& Schüssler, M. 2007, A\&A, 465, L43

Wang, Y. M., Lean, J. L., \& Sheeley, N. R. 2005, ApJ, 625, 522

Wenzler, T., Solanki, S. K., Krivova, N. A., \& Fröhlich, C. 2006, A\&A, 460, 583

Wu, C. J., Usoskin, I. G., Krivova, N., et al. 2018a, A\&A, 615, A93

Wu, C.-J., Krivova, N. A., Solanki, S. K., \& Usoskin, I. G. 2018b, A\&A, 620, A120

Yeo, K. L., Solanki, S. K., Krivova, N. A., et al. 2020, Geophys. Res. Lett., 47

Zwaan, C. 1978, Sol. Phys., 60, 213 